\title{
Mixing characterization in different helically coiled configurations by laser-induced fluorescence
}

\author{
P. Kováts ${ }^{1} \cdot$ C. Velten ${ }^{1} \cdot$ M. Mansour ${ }^{1,2} \cdot$ D. Thévenin ${ }^{1} \cdot K_{\text {K. Zähringer }}^{1}$ (D)
}

Received: 5 June 2020 / Revised: 30 July 2020 / Accepted: 14 August 2020

(c) The Author(s) 2020

\begin{abstract}
Flow Mixing of two miscible liquids has been characterized experimentally in three different helically coiled reactor configurations of two different lengths in the laminar flow regime at $R e=50 \ldots 1000$. A straight helical coil, a coiled flow inverter, and a new coiled flow reverser have been built, each in a 3-turn and a 6-turn configuration. Laser-induced fluorescence of resorufin has been used to visualize and quantify mixing in cross-sections throughout the reactors. A mixing coefficient is derived from the fluorescence images to allow for a quantitative measure and comparison of the six configurations. It becomes obvious from these experimental results, that an early flow redirection in the helical configuration is beneficial to mixing. The 3-turn reactors achieve nearly the same mixing coefficients as the 6-turn reactors with the double length. This can be explained by the stabilizing effect of the Dean vortices in the helix, which develop during the first two turns. After that, the liquid is trapped inside the vortices and further mixing is inhibited. Accordingly, the coiled flow inverter and coiled flow reverser configurations lead to much higher mixing coefficients than the straight helical coil. The results of these measurements are now used for validation of numerical simulations, which reproduce the geometrical and flow conditions of the experiments. Some exemplary results of these calculations are also shown in this article.
\end{abstract}

K. Zähringer

katharina.zaehringer@ovgu.de

1 Lehrstuhl für Strömungsmechanik und Strömungstechnik, Otto-von-Guericke-Universität Magdeburg, 39106 Magdeburg, Germany

2 Mechanical Power Engineering Department, Faculty of Engineering, Mattaria, Helwan University, Cairo 11718, Egypt 


\section{Graphic abstract}

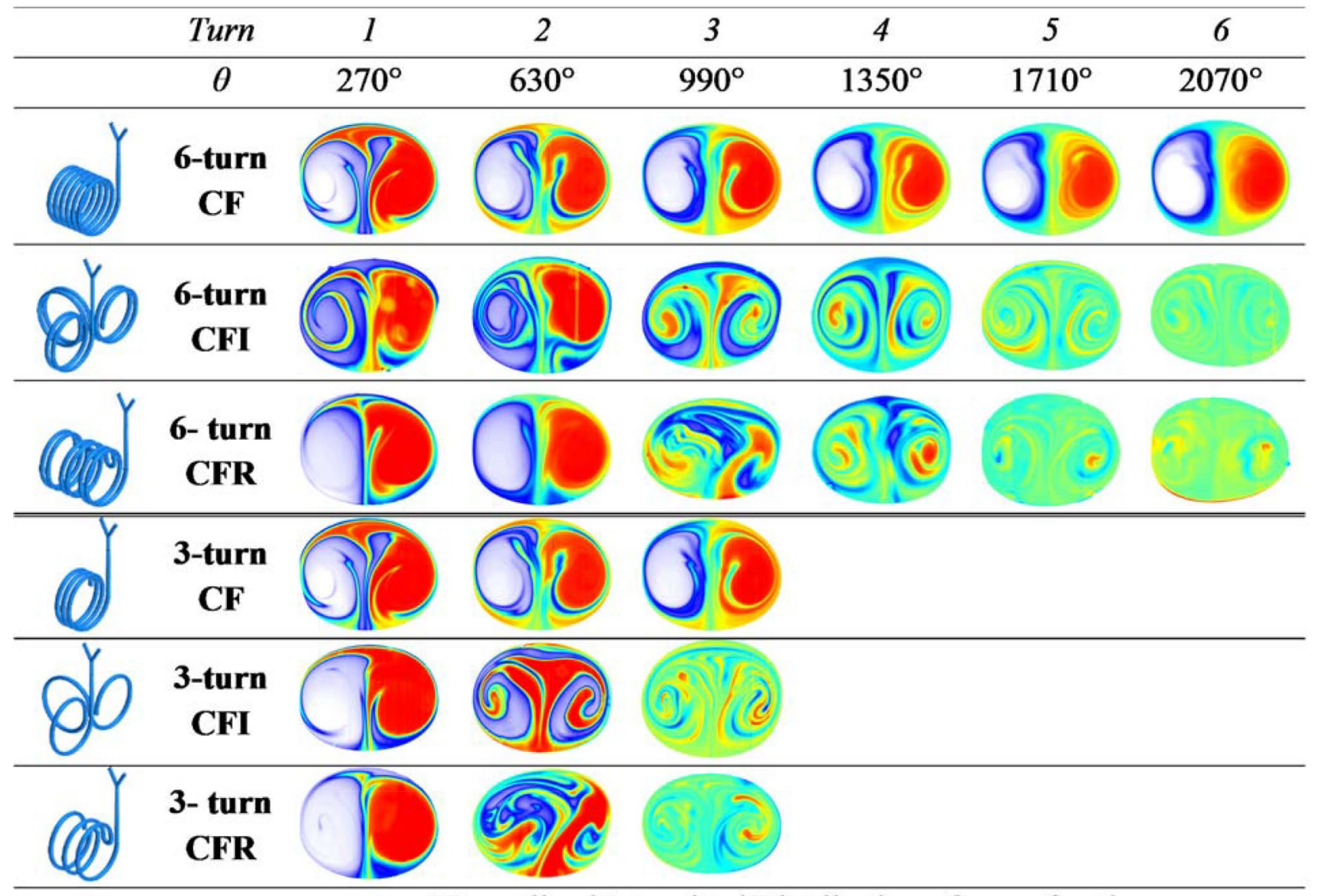

\section{Normalized Intensity / Distribution of mass fraction}

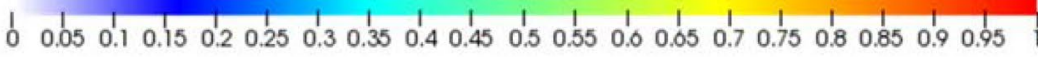

Mass fractions of tracer fluid at $R e=500$ in the six examined helix configurations.

\section{List of symbols}

A Cross-sectional area $\left(\mathrm{m}^{2}\right)$

$A_{f} \quad$ Area of a grid unit $\left(\mathrm{m}^{2}\right)$

$A_{\text {Pixel }}$ Pixel area $\left(\mathrm{m}^{2}\right)$

$c \quad$ Local concentration $\left(\mathrm{kg} / \mathrm{m}^{3}\right)$

$\bar{c} \quad$ Average concentration $\left(\mathrm{kg} / \mathrm{m}^{3}\right)$

CF Straight standard helix

CFD Computational fluid dynamics

CFI Coiled flow inverter

CFR Coiled flow reverser

$d \quad$ Tube diameter $(\mathrm{m})$

$D \quad$ Coil diameter (m)

$\mathfrak{D}_{a b} \quad$ Mass diffusivity $\left(\mathrm{m}^{2} / \mathrm{s}\right)$

De Dean number (-)

FEP Fluorinated ethylene propylene

$i \quad$ Total coil number (-)

I Intensity (counts)
$\bar{I} \quad$ Normalized intensities (-)

$I_{0} \quad$ Minimum intensity (counts)

$I_{\mathrm{AVG}} \quad$ Average Intensity (counts)

$I_{\max } \quad$ Maximum intensity (counts)

$L \quad$ Total coil length (m)

L1 Liquid 1

L2 Liquid 2

PLIF Planar laser-induced fluorescence

$M_{c} \quad$ Mixing coefficient (-)

$n \quad$ Refractive index (-)

$P \quad$ Coil pitch (m)

$Q \quad$ Volume flow rate $\left(\mathrm{m}^{3} / \mathrm{s}\right)$

Re Reynolds number (-)

$s \quad$ Wall thickness (m)

Sc $\quad$ Schmidt number (-)

$u \quad$ Mean velocity $(\mathrm{m} / \mathrm{s})$ 


\section{Greek symbols}

$\delta \quad$ Curvature ratio (-)

$\eta \quad$ Dynamic viscosity $(\mathrm{Pa} \cdot \mathrm{s})$

$\theta \quad$ Angle of revolution $\left({ }^{\circ}\right)$

$\rho \quad$ Fluid density $\left(\mathrm{kg} / \mathrm{m}^{3}\right)$

\section{Introduction}

Coiled helical reactors are used in industrial processes for static mixing or heat and mass transfer to intensify these operations compared to straight tubular reactors (Vashisth et al. 2008). The development of secondary flows (Dean vortices) in these helical tubes enhances the radial mixing, while keeping a low axial back-mixing behaviour. This increases heat and mass transfer and leads to narrower residence time distributions.

Although numerous studies were previously conducted to investigate the influence of different parameters on the advantages of helical pipes concerning heat and mass transfer (Gelfgat et al. 2003; Kockmann 2020; Kurt et al. 2015a, b; Kushwaha et al. 2020; Liu et al. 2005; Mei et al. 2020; Mori and Nakayama 1965; Moulin et al. 1996; Naphon and Wongwises 2006; Rainieri et al. 2012; Rennie and Raghavan 2005; Schmidt 1967; Sedahmed et al. 1985; Tohidi et al. 2015; Yasuo and Wataru 1965), the characterization of pure mixing of miscible liquids in such apparatus, which is the fundament of the aforementioned transfer processes, has rarely been undertaken. The group of Nigam (Kumar et al. 2006; Sharma et al. 2017) investigated experimentally and numerically the influence of Reynolds number $\operatorname{Re}=(u \cdot d \cdot \rho) / \eta$, Schmidt number $S c=\eta /\left(\rho \cdot \mathfrak{D}_{a b}\right)$, and curvature ratio $\delta$ on the mixing performances of different coiled geometries, by determination of the residence time. In these equations, $u$ is the mean velocity in the tube, $\rho$ is the fluid density, $\eta$ is the dynamic viscosity, the curvature ratio $\delta$ is defined as the ratio of the tube diameter $d$ to the coil diameter $D, \mathfrak{D}_{a b}$ is the mass diffusivity from the first fluid into the second fluid. They conclude that mixing increases with the Reynolds number, while Schmidt number only plays a role for rather low Reynolds-numbers, $\operatorname{Re}<20$.

A systematic investigation of these parameters, also including Dean number $D e=R e \cdot \sqrt{\delta}$, was executed numerically in (Mansour et al. 2019, 2020b). It was also found that the influence of $S c$ is only important for very low Reynolds number $(R e<40)$, where mixing is better for lower $S c$, i.e. increased diffusion. For $R e \geq 40$, the secondary flow is strong enough and mixing is dominated only by flow convection, where the effect of Schmidt number becomes negligible. It was also shown that the increase in the curvature ratio $\delta$ leads to stronger secondary flows and better mixing, which is more pronounced at low Reynolds numbers. Additionally, it was shown that the increase of the coil pitch $P$ is generally negative concerning mixing and it should be kept minimum. However, at very low Reynolds number (very weak secondary flows), a higher pitch improves mixing due to the increased torsion (flow twisting). Previous numerical studies of the authors showed further, that there are two optimal values of the Reynolds number, leading to the highest possible mixing in helical pipes at around $R e \approx 35-50$ and $R e \approx 650-1000$ (Mansour et al. 2019, 2020b). Here, it should be noted that the pressure drop in a helically coiled tube is generally higher compared to a straight pipe of the same length (Ito 1959; Mishra and Gupta 1979). Accordingly, the first optimal Reynolds number should normally be preferred since the pressure drop is significantly lower compared to the second optimal value.

In the literature, numerous other coiled structures were also employed to improve mixing, starting from simple modifications of the cross-section profile (Dong and Shufen 2014; Jiang et al. 2004; Schönfeld and Hardt 2004; Vanka et al. 2004), passing through significant changes in the flow direction (Lasbet et al. 2007). Additionally, more complex variations were introduced, such as corrugated helical pipes (Ndiaye 2017; Rainieri et al. 2012; Zachár 2010). Further, composite and even more complicated coiled configurations combining several structures were also used (Alam and Kim 2012; Castelain and Legentilhomme 2006; Hajmohammadi et al. 2013; Tohidi et al. 2015; Wang et al. 2018). Nevertheless, most of these configurations involve a considerable increase in pressure drop, preventing their practical implementation.

Saxena and Nigam (Saxena and Nigam 1984) introduced a structured configuration called the coiled flow inverter (CFI), in which the flow direction is inverted by bending the coils. In this way, the direction of the secondary flow is changed in order to improve mixing. The CFI can be simply constructed by fitting $90^{\circ}$ bends at regular length intervals between the coils and it attracted, also due to its geometrical compactness, some more interest for industrial applications. However, the CFI performance was found to be comparable to a simple, straight coil, if the number of inversions is limited (Kumar et al. 2007; Mridha and Nigam 2008a, b). Therefore, numerous flow inversions are always needed for a significant mixing enhancement. Additionally, the flow is usually inverted after at least 3-4 turns in most CFI applications, to ensure fully developed vortices before changing the flow direction (Kumar et al. 2007; Mandal et al. 2011; Mridha and Nigam 2008a, b; Saxena and Nigam 1984). Consequently, a considerable increase in pressure drop is unavoidable in most cases. Nevertheless, it was recently shown that mixing can even be improved by early flow inversion in the entrance region (Khot et al. 2019; Mansour et al. 2017). Therefore, the flow path of a straight helical pipe should be altered before the flow gets fully developed. Accordingly, mixing can be significantly improved with only a limited 
number of flow re-directions (for instance two) without any remarkable increase in pressure drop.

A novel coiled configuration called the coiled flow reverser (CFR) was introduced in (Mansour et al. 2020c), by alternatively reversing the coiling direction after one or two turns. In this configuration, the flow direction is completely reversed, creating a longer developing region, and a more complex secondary flow. Mixing of two miscible liquids in the CFR, the CFI, and the straight coil was numerically studied and compared in that publication, considering different flow conditions and two inlet conditions, i.e. a parallel and a perpendicular interface to the coil axis between the two liquids at the inlet. It was shown that the performances of the CFI and the CFR are comparable and obviously higher than those of the straight coil, particularly for the perpendicular interface at the inlet, that leads to very bad mixing in the straight coil. Further, at high Reynolds number (Re > 500), mixing and heat transfer in the CFR was found more efficient than in the straight coil and CFI. Nevertheless, the whole study was performed by numerical simulations and simplified inlet conditions, which cannot be achieved experimentally.

With this in mind, the focus of the present work is to validate the findings of (Mansour et al. 2020c) experimentally and to further compare the mixing behaviour of two miscible liquids within these different coiled configurations in the laminar flow regime. Laser-induced fluorescence is used here for experimental mixing characterization. The coiled geometries are used in two different lengths, with 3 and 6 coils. Half of the liquid flowing through the coils is marked by the fluorescent tracer resorufin, thus allowing for a locally resolved assessment of the concentrations and thus mixing in the coils. The experimental results are completed by exemplary CFD calculations that are validated by the experiments and represent now an easy-to-apply tool for further mixing studies in helical reactors.

\section{Choice of flow conditions and helix geometries}

The flow conditions leading to the apparition of secondary flow and symmetric vortices can be described by the Dean number, which shows a dependency on the curvature ratio $\delta$ and the Reynolds number. The latter influences the complex behaviour of flow mixing in helical coils and was studied and discussed in previous investigations as presented in the introduction. In fact, mixing shows a non-monotonic behaviour with the change of Reynolds number, since the residence time, the vortex structure, and the vortex strength change simultaneously with $R e$. This can be explained by Fig. 1, which presents the vortex structures as a function of Reynolds number for a sample straight helical pipe taken from (Mansour et al. 2017). This sample helical pipe has a tube diameter of $d=10 \mathrm{~mm}$, a coil diameter of $D=118$, amd a pitch of $P=16 \mathrm{~mm}$. As shown in Fig. 1 left, for very low Reynolds number $(R e \leq 35)$, the secondary flow is very weak. In this range, increasing the Reynolds number rapidly strengthens the Dean vortices, thus noticeably enhancing mixing, which leads to a first mixing maximum at $R e \approx$ 40. For $40<\operatorname{Re}<200$, mixing decreases due to the slight decrease in residence time together with the very strong vortices, which trap the liquids and prevent the evolution of mixing. Then, flow mixing increases again for $R e>200$ up to a second maximum approximately at $R e \approx 650-1000$, due to the continuous increase in the secondary flow strength and the improved vortex structure. Afterwards (Fig. 1, right), the mixing decreases monotonically as a result of the strongly decreased residence time (Sharma et al. 2017) and the poor vortex structure, where one vortex diminishes while the second one is strongly tilted (Mansour et al. 2017). Starting with Reynolds numbers greater than 1000 , also the transitional flow regime starts (Cioncolini and Santini 2006; Sharma et al. 2017). For this reason and also due to the fact

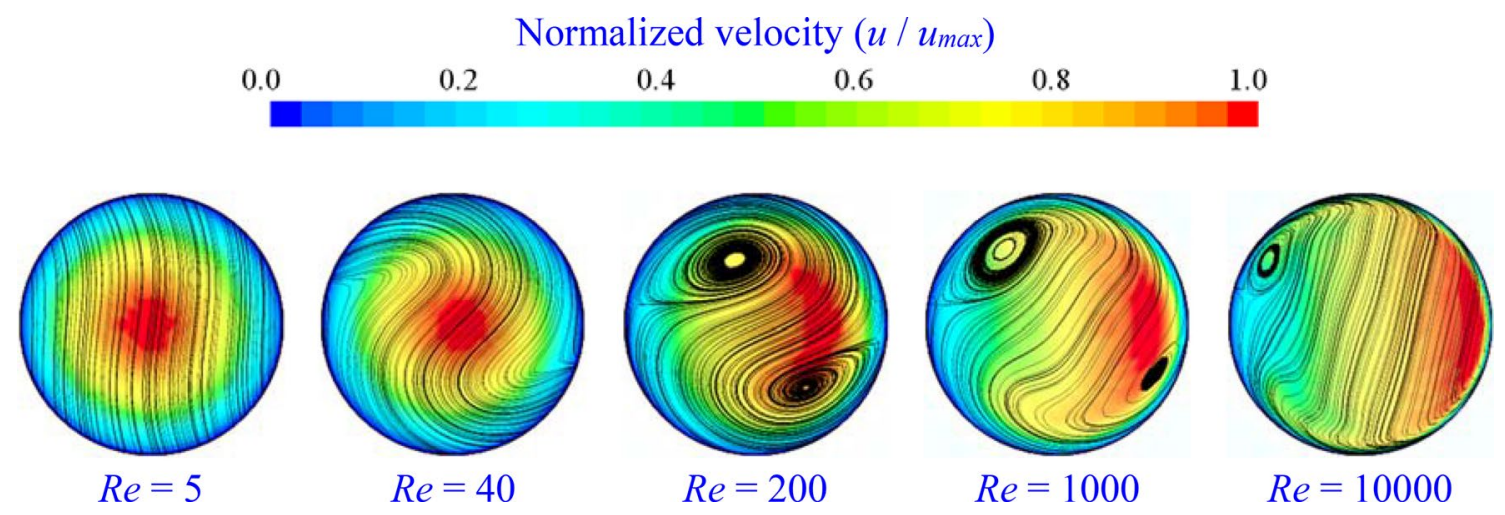

Fig. 1 Secondary flow structures for different Reynolds numbers at the outlet of a 5-turn straight helical pipe. Calculations from (Mansour et al. 2017) 
that one main application of coiled mixers lies in the laminar flow regime, this study only considers Reynolds numbers from $50 \leq R e \leq 1000$.

It has also been shown in previous studies, that the Dean vortices in helical coils are already fully developed in the first 2-3 turns (Austin and Seader 1973; Kováts et al. 2018; Mansour et al. 2018; Saffari and Moosavi 2014; Vashisth and Nigam 2009). This leads in most cases to a slow improvement of flow mixing in later turns, since the fluid is trapped in the two vortices. This can be avoided by inverting the flow direction in the coil, which induces a new developing vortex pair, that causes a significant increase in mixing, heat and mass transfer (Khot et al. 2019; López-Guajardo et al. 2017; Saxena and Nigam 1984). This flow inversion can be provided by the use of a coiled flow inverter (CFI), which reverts the flow by $90^{\circ}$ and a novel geometry, called coiled flow reverser (CFR) (Mansour et al. 2020c), which leads to a $180^{\circ}$ flow reversion.

These two geometries have been used in this study, together with a straight, horizontal coil for comparison. In each case, two different lengths, with a total number of $i=6$ or $i=3$ coils, are investigated. In the case of the CFI, the flow is diverted by two $90^{\circ}$ bends, in the CFR two $180^{\circ}$ reversals are realized. Bends and reversals are introduced after the 2 nd and 4th coil for the 6-turn configuration and after the 1st and 2nd coil for the 3-turn configuration. The different models of these helical reactors are shown in Fig. 2.

The helical reactors were made of FEP-pipe (fluorinated ethylene propylene) with an inner pipe diameter of $d=10 \mathrm{~mm}$, a wall thickness $s=1 \mathrm{~mm}$, an inner coil diameter of $D=118 \mathrm{~mm}$ and a coil pitch of $P=16 \mathrm{~mm}$. Considering the present dimensions, the coil curvature ratio resulted in $\delta=d / D=0.0847$ and the total coil length $L=i \cdot \sqrt{\pi^{2} D^{2}+P^{2}}=2.23 \mathrm{~m}$ for 6 -turn and $1.11 \mathrm{~m}$ for 3 -turn configuration.

The FEP-pipe offers the required properties for optical measurements that are transparency and a refractive index close to that of water. Due to its high rigidity, it is difficult to adapt the pipe to the needed coil diameter. Therefore, it is treated by thermal deformation. To reduce the risk of kinks, the pipe is filled with water and heated up to $80^{\circ} \mathrm{C}$ in a drying oven. Subsequently, the pipe can be wound clockwise and fixed on a PVC core of the appropriate form and diameter. The straight standard helix $(\mathrm{CF})$ is only produced in the 6-turn configuration, as the measurement results after the first 3-turns can be used directly for comparison with the other 3-turn configurations. The realization of the bending of the CFI is carried out using a sleeve, whereby the pipes can be rotated against each other. Accordingly, the coils are cut and attached individually for each arm of the helix, all with a clockwise winding. For the CFR, 3D-printed $180^{\circ}$
Fig. 2 Geometry of the 6 different helix configurations examined in this study

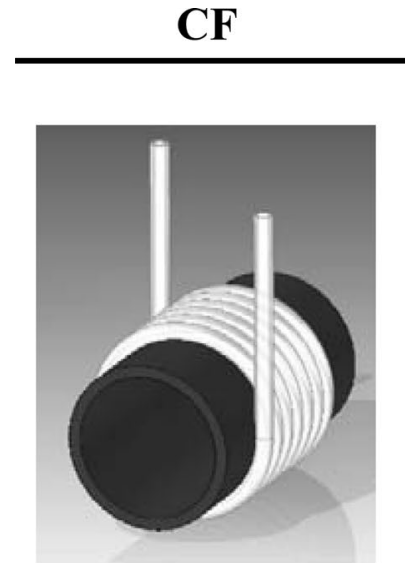

CFI

CFR

\section{6-turn configurations}
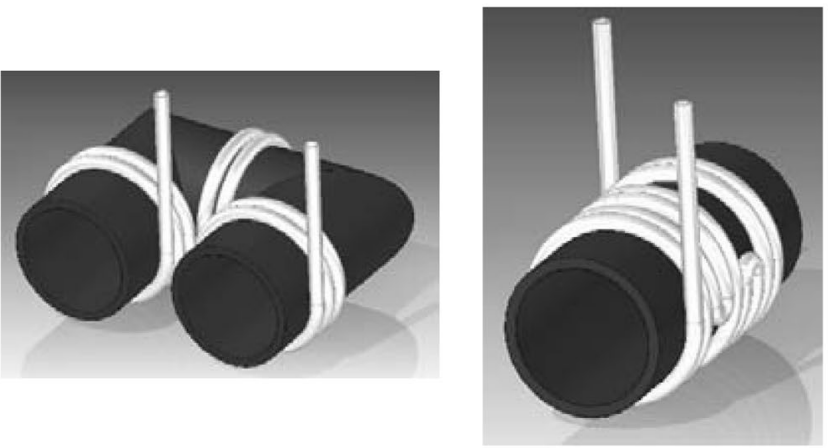

$\underline{\text { 3-turn configurations }}$

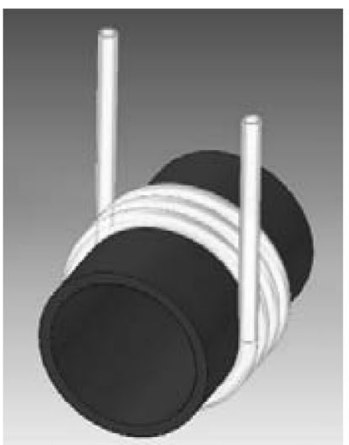

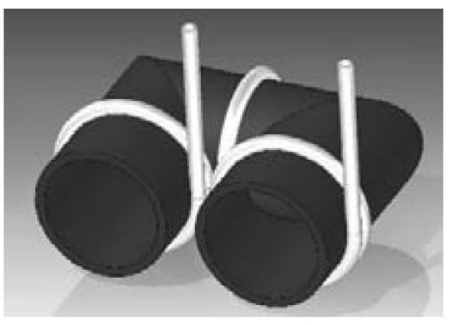

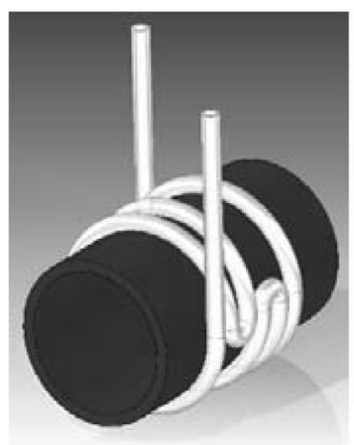


reversers are used. Between the reversers, the winding has to be reversed to the counter-clockwise direction. These technical realizations are shown in Fig. 3.

For all reactors, measurements were carried out in the range of laminar flow regimes with $50 \leq R e \leq 1000$. The resulting Dean numbers and total volume flow rates $Q=\frac{\pi \cdot d \cdot \eta \cdot R e}{4 \cdot \rho}$ are shown in Table 1 .

The mixing efficiency of the two-component system is investigated by using two fluids, deionized water, and a solution of deionized water and resorufin $(1 \mathrm{mg} / \mathrm{L} \pm 1 \%$, CAS: 34994-50-8, Sigma-Aldrich), which is used as a tracer dye for the PLIF-measurements. Both solutions are set to the same flow rate (accuracy $\pm 0.04 \mathrm{ml} / \mathrm{s}$ ), which is thus half of the total flow rates given in Table 1, and introduced to the set-up via a Y-connector. Because of the low resorufin concentration, its influence on physical properties can, as verified by measurements with a Malvern Kinexus pro + rotational rheometer before, be neglected. Therefore, all calculations are based on the corresponding physical properties of water for standard conditions at $20{ }^{\circ} \mathrm{C}$ in the air-conditioned room $\left(\rho=998.23 \mathrm{~kg} / \mathrm{m}^{3}, \eta=0.001003 \mathrm{~Pa} \cdot \mathrm{s}\right.$ (VDI 2013)).

\section{Experimental setup}

\subsection{Refractive index matching}

For the use of optical measurement techniques in complex geometries like the helical coils examined in this study, it is essential to minimize optical distortions, due to different refractive indices of working fluid, wall material, and surrounding fluid. To this end, the reactors are placed in a rectangular tank that is filled with a liquid with the same refractive index as the FEP-pipe. Ideally, also the working fluid would have a matched refractive index, but this was not
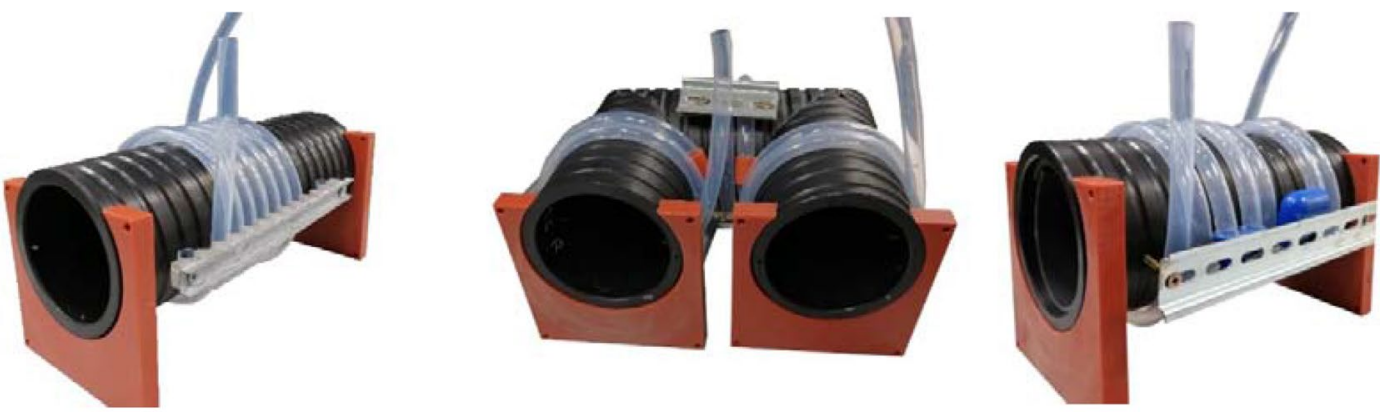

\section{$\underline{\text { 3-turn configurations }}$}

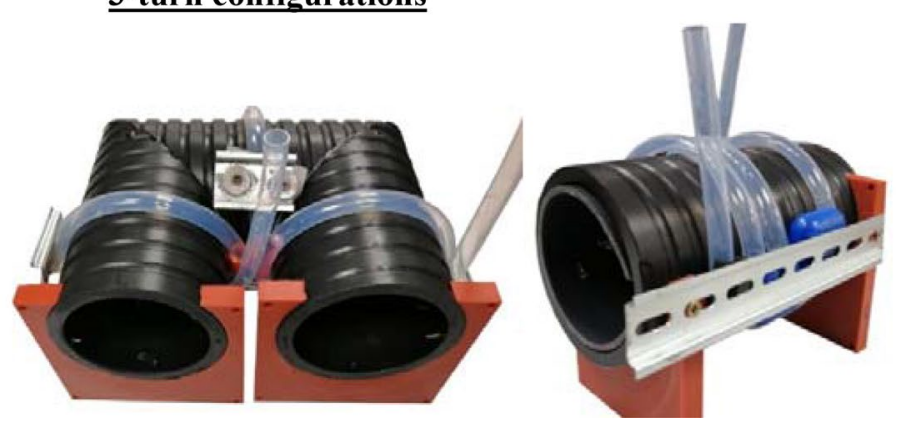

Fig. 3 Technical realizations of the different reactor geometries

Table 1 Flow conditions

\begin{tabular}{lllllll}
\hline Reynolds number $R e$ & {$[-]$} & 50 & 100 & 200 & 500 & 1000 \\
Dean number $D e$ & {$[-]$} & 15 & 31 & 61 & 154 & 307 \\
Flow rate $Q$ & {$[\mathrm{~mL} / \mathrm{min}]$} & 24 & 48 & 94 & 236 & 472 \\
\hline
\end{tabular}




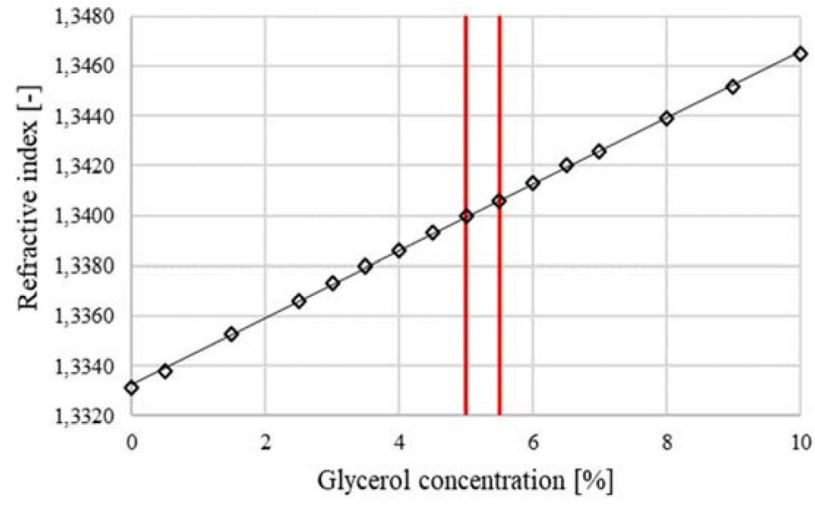

Fig. 4 Refractive index as a function of the volumetric glycerol concentration in water

possible here, due to the incompatibilities of the materials inside the set-up.

The refractive index of the FEP-pipe varies slightly for production reasons. Therefore, it was determined by gradual addition of glycerol into distilled water. The measured linear dependency of the refractive index on the volumetric glycerol concentration (accuracy $\pm 0.1 \mathrm{vol} \%$ ) is shown in Fig. 4 . The refractive index of the glycerol-water solution was then measured with a refractometer (Abbemat 200 from Anton Paar, accuracy $\pm 0.000100 \mathrm{nD}$ ) for each step and an image was taken of the pipe in front of a line and checkerboard pattern in the solution. Figure 5 shows examples of these images. If the refractive index is not matched (top image of Fig. 5), the background pattern shows strong distortions at the position of the tube wall. On the other hand, if the refractive index of the pipe is the same as the one of the glycerol-water solution (centre and bottom images of Fig. 5), the pipe becomes nearly invisible in the solution. This is the case for a glycerol concentration of 5-5.5 vol\% (red vertical lines in Fig. 4) which corresponds to a refractive index of $n=1.3400 \ldots 1.3406$.

\subsection{Experimental setup}

Figure 6 shows the main components of the experimental setup. The helical reactor $(\mathrm{H})$ is fixed in a rectangular tank made of acrylic glass and filled with the 5 vol\% glycerol solution to match the refractive index. The helical reactor has a Y-inlet connector $(\mathrm{Y})$ which provides the equal inlet flow of the two solutions. These are provided gravity-driven from two storage tanks which are situated about $1.5 \mathrm{~m}$ above the reactor. For flow regulation, needle valves are used on each side. Note that the orientation of the Y-inlet connector with respect to the coil axis affects the mixing performance significantly (Mansour et al. 2017). The best orientation of the Y-inlet connector is when the interface of the two liquids at the inlet is parallel to the coil axis. In this case, the
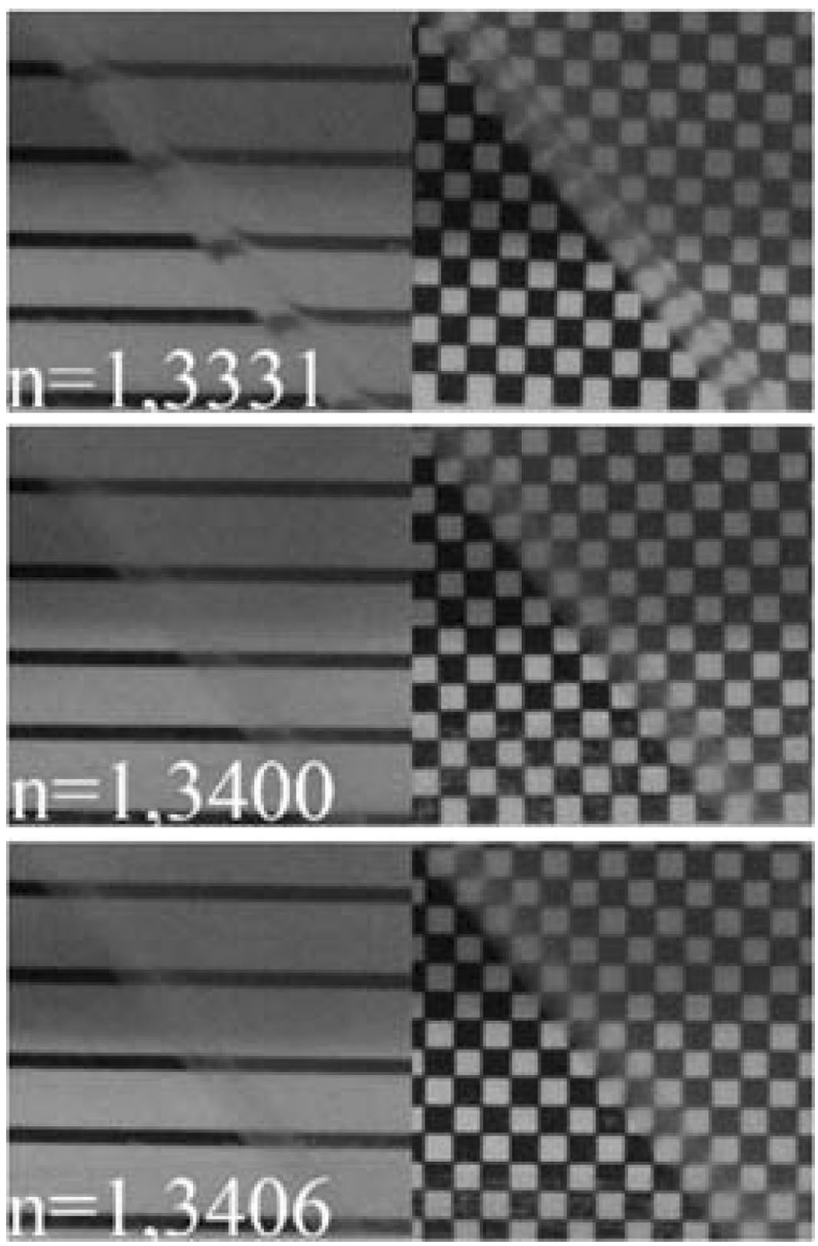

Fig. 5 Determination of the refractive index of the FEP-pipe

generated secondary vortices are optimally perpendicular to the inlet interface, leading to the highest possible mixing. On the other hand, the worst case would be when the interface of the two liquids at the inlet is orthogonal to the axis of the coiled reactor, in which one vortex appears in each liquid, reducing the mixing speed. In the present work, the worst case was considered, i.e. the orientation of the Y-inlet connector is fixed in a way that the separation of the two liquids is orthogonal to the axis of the reactor. This position has been chosen to allow for the best discrimination between the different reactors examined.

The fluorescent dye resorufin is used for the visualization of mixing in the top cross-sections of the different reactors. Fluorescence of resorufin is excited by a Nd:YAG-laser (L) at $532 \mathrm{~nm}$ with $15 \mathrm{~mJ} / \mathrm{p}$ and formed to a light sheet $(\mathrm{O})$ that traverses the top reactor tubes perpendicularly (see Fig. 6). Fluorescence intensities are detected at $90^{\circ}$ to the light sheet with a LaVision Imager sCMOS camera (C) with $2560 \times 2160$ pixels, and using a Tokina macro $100 \mathrm{~mm}$ objective with a $555 \mathrm{~nm}$ high pass filter. The field of view of the light sheet and the camera is adjusted to the size of one 


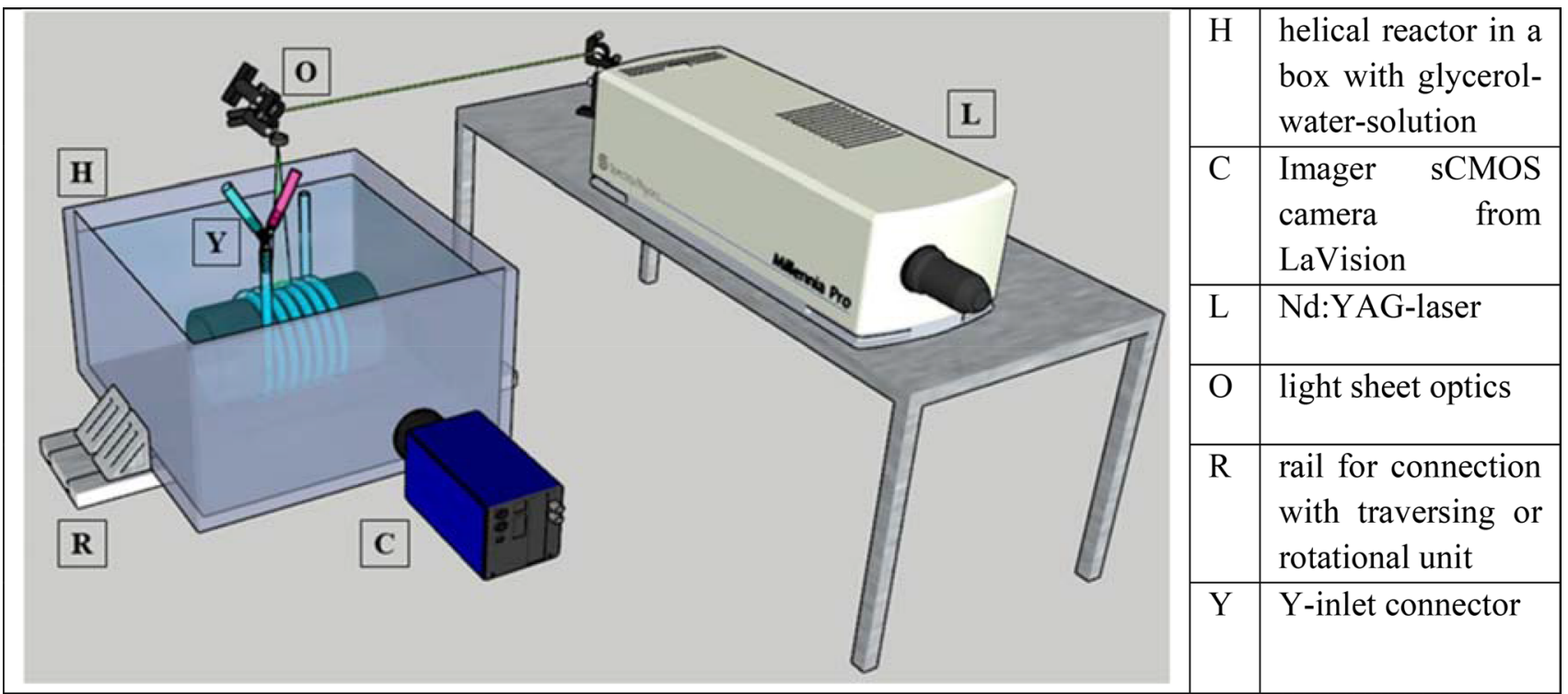

Fig. 6 Main components of the experimental setup

coil, to keep laser energy variations low in the considered region. The whole tank, including the reactor, is successively moved to all other, 3 or 6 , coil positions, without moving the optical set-up. The adjustment of the measuring position is realized by the use of a traversing unit for the $\mathrm{CF}$ and $\mathrm{CFR}$ or a rotation unit for the CFI (R).

For each position, 200 images with a recording frequency of $5 \mathrm{~Hz}$ are taken. This allowed for a mean image with only $<7 \%$ standard deviation. Three different types of images had to be recorded: first, the system is filled with deionized water to obtain images with the minimum intensity for background correction (Fig. 8, centre, left image); second, the $1 \mathrm{mg} / \mathrm{L}$ resorufin solution is filled into the coils to get images with the maximum fluorescence intensity for light sheet correction (Fig. 8, top, left image), and then the measurement images could be acquired (Fig. 8, bottom, left image). For this, the volume flow rate is adjusted manually for each storage tank to half of the flow rate given in Table 1 . After considering the time that is needed for the full development of the flow in the helical reactor, images are taken. Control of laser and camera is done by the software DaVis 8.4.0 and a synchronization unit. Additionally, laser energy variations were acquired on an image-by-image basis and used for automatic image intensity correction.

\section{Postprocessing of experimental data}

Image treatment with the software DaVis 8.4. is the first of two steps for post-processing the obtained experimental data. Hence, the first operation is to create an average of the 200 measuring images, which provide the intensity $I$, mean background images with the minimum intensity $I_{0}$, and mean sheet images with the maximum intensity $I_{\max }$. Subsequently, a normalization is done for each mean intensity image, which provides background and sheet correction according to the following equation:

$\bar{I}=\frac{I-I_{0}}{I_{\max }-I_{0}}$.

This leads to normalized intensities $I$ in the range between 0 and 1 . These intensities correlate linearly with the local concentration of resorufin in the cross-section, since resorufin fluorescence has a linear relationship with the concentration in the considered range $(<1 \mathrm{mg} / \mathrm{L})$ (Kováts and Zähringer 2018 and Fig. 7). This means that the explicit calculation of concentrations is not necessary for this study,

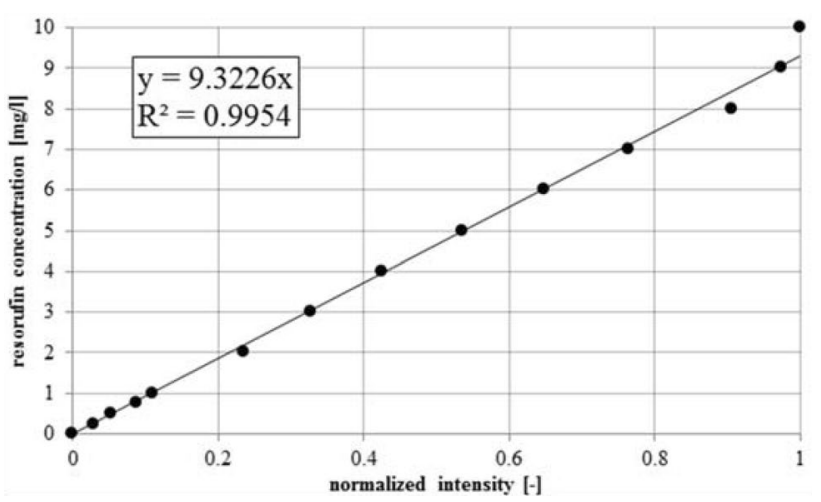

Fig. 7 Calibration curve for resorufin 
Fig. 8 Steps of image treatment for preparation of the calculation of mixing coefficients

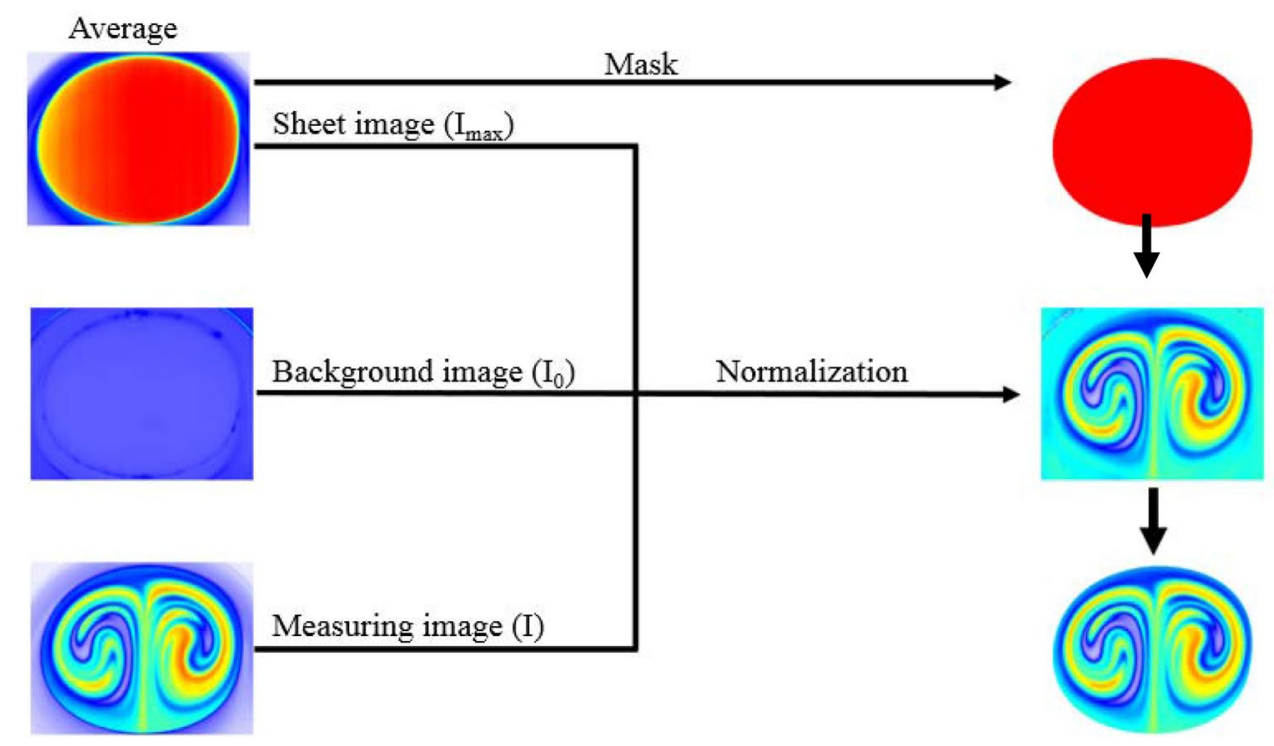

since the intensities will be further processed to calculate mixing coefficients (see below).

In the last step, a mask is created from the mean sheet images (maximum intensity) by binarizing the intensity of the liquid and the inner pipe wall. After the application of the mask, the images can be used for the calculation of mixing coefficients in MATLAB. Considering the fact, that both solutions (pure water and resorufin solution) have the same flow rate and the concentration is linear with the fluorescence intensity, these normalized intensity images, correspond directly to the mass fraction of resorufin. A summary of these essential processing steps is given in Fig. 8.

To assess the quality of mixing, the mixing coefficient $M_{c}$ is calculated by use of the following equation:

$M_{c}=1-\frac{\sum_{f}|c-\bar{c}| A_{f}}{|\bar{c}| \sum_{f} A_{f}}$ with $\bar{c}=\frac{1}{A} \int c d A$,

where $c$ is the local concentration of a component, $\bar{c}$ the average concentration over the cross-sectional area $A$, and $A_{f}$ is the area of a grid unit. The mixing coefficient is in the range from 0 to 1 , where 0 shows the case of no mixing, and 1 refers to perfect mixing (Mansour et al. 2017). It is based on the surface-weighted averaging of the mass fraction. The calculation of the mixing coefficients $M_{c}$ for the experimental part of this study is based on the fluorescence intensity values. For this, Eq. (2) has to be adapted accordingly:

$M_{c}=1-\frac{\sum\left|I-I_{\mathrm{AVG}}\right| \cdot A_{\text {Pixel }}}{I_{A \mathrm{VG}} \cdot \sum A_{\text {Pixel }}}$.

Here, $I$ is the intensity of each pixel, $I_{\mathrm{AVG}}$ describes the average intensity of the cross section and $A_{\text {Pixel }}$ is the area of a pixel. Given the fact, that the intensities of the background, sheet and measurement images could be determined with a standard deviation of better than $4 \%, 6 \%$, and $7 \%$, respectively, the accuracy of the calculated mixing coefficient lies in the order of $10 \%$.

\section{Experimental results and discussion}

The experimental results for the mass fraction distribution, obtained in the 6-turn and 3-turn coils at all examined Reynolds numbers, are shown in Fig. 9. The results show the fields of mass fraction in the cross sections on top of the coils that are crossed by the laser light sheet. Since the entrance to the coils is situated on the front side (see Fig. 3) of the set-up, the first cross section is obtained at $3 / 4$ of a turn, that means $\theta=270^{\circ}$, if following the coil in the streamwise direction. The following cross sections are situated always one turn, which means $360^{\circ}$ after.

In the first row of each plate on this figure the results for the 6-turn straight helical coil are represented. It can be seen, that two Dean vortices are stabilized in this straight coil, that develop during the first three turns and remain nearly unchanged in the second half of the helix. These vortices trap the two liquids, that are pure water (white to blue colour in Fig. 9) and resorufin solution (orange to red colour in Fig. 9), and very poor mixing is obtained in that way. Since both solutions are injected with the same flow rate, ideal mixing would lead to a mass fraction of 0.5 , which is shown by turquoise colour in the following representations. With growing Reynolds number, the two vortices become more unstable and some spiral lamellas appear at $R e=500$.

Due to the bending of the flow in the CFI and CFR, the aforementioned stable vortices have to rearrange after each 


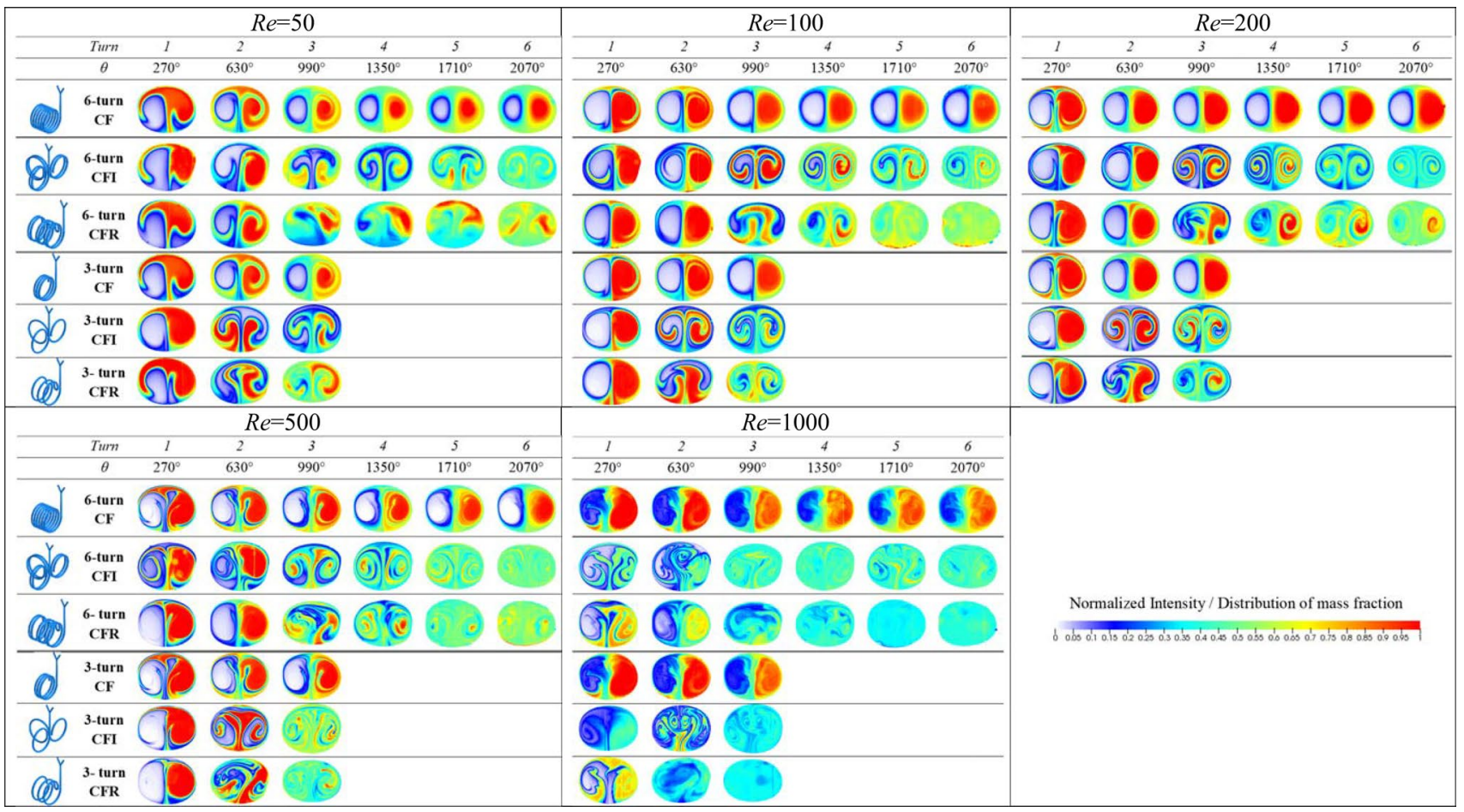

Fig. 9 Mass fractions obtained by experiments for all configurations and Reynolds numbers

change of the flow direction (between 2nd and 3rd; 4th and 5 th turn for the 6-turn configurations and between each turn for the 3-turn configurations). This is visible in Fig. 9 (2nd and 3rd row of each plate) by an increased flow complexity and thus mixing of the two liquids. Regions with complete mixing (mass fraction $=0.5$; turquoise colour) increase distinctly after each redirection. This effect is more pronounced for the CFR than for the CFI at $R e<200$. At $R e=500$ and $R e=1000$ this difference disappears and both reactors end up with nearly the same mixing intensity. These effects are valid for both, the 6-turn and the 3-turn configurations.

It is further worthwhile to compare the 6-turn (first three rows of each plate) with the 3-turn configurations (last three rows of each plate). Especially for the higher Reynolds numbers $(R e \geq 500)$, the differences between the last turn of both seem rather small in all three coil configurations. Only for the $R e \leq 200$, the last turn of the 6-turn CFI and CFR looks better mixed than the last turn of the 3-turn configuration. This shows that the very early flow redirection in the 3-turn configurations is obviously beneficial to good mixing results. The 3-turn configurations of CFI and CFR show much better mixing after 3-turns than after the same length in the 6-turn configurations. These had, up to the third turn, only one flow redirection, contrary to the 3-turn configuration with two changes in the flow direction.

For $R e=1000$ a supplementary vortex structure can be recognized, especially in the case of the 3-turn CFI, in the upper centre of the image showing the 2nd and 3rd turns (Fig. 9). These "Lyne-Vortices" appear from time to time at around $R e \geq 700$ in all configurations, but are stabilized in the case of the CFI and thus visible on the mean images. A study discerning the conditions of their development and their influence on the mixing behaviour is under work.

The calculated mixing coefficients are represented at all Reynolds numbers in Fig. 10 and Fig. 11 for the 6-turn and 3-turn configurations respectively. It is obvious from these Figures, that the configurations with redirection of the flow (CFI and CFR) exhibit much higher mixing coefficients at the outlet of the reactors, than the straight helix. This increase in the mixing coefficient starts with the first redirection of the flow and is always clearly apparent after the third turn by a steeper increase of the curves. The mixing coefficients of the straight coil stay rather constant after 2-3 turns. Further, it can be recognized, that even if considering the measurement accuracy of around $10 \%$, the CFR is most often the best mixing reactor at the outlet after 6 or 3 turns respectively. The comparison of the mixing coefficients of the 3-turn and 6-turn configurations also confirms, that at higher Reynolds number, both lengths lead to nearly complete mixing, thus confirming once more the importance of early flow inversion. 


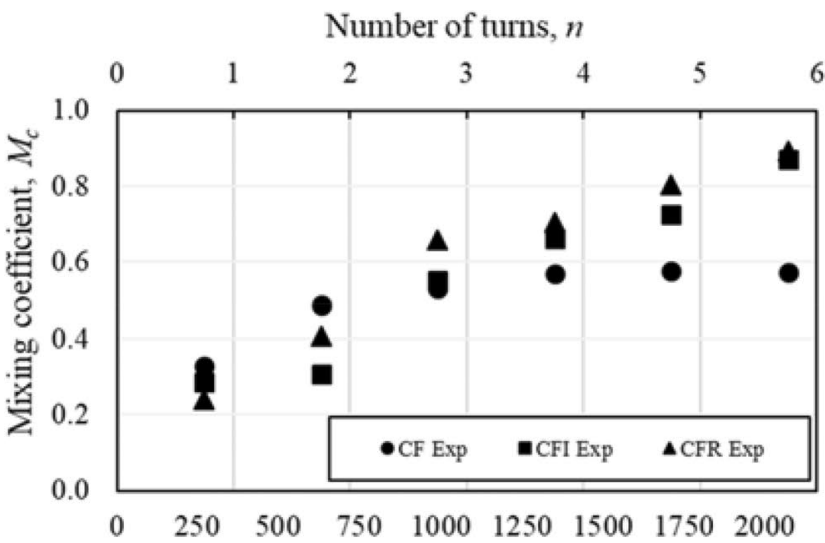

Angle of revolution, $\theta\left[^{\circ}\right]$

$$
R e=50
$$

Number of turns, $n$

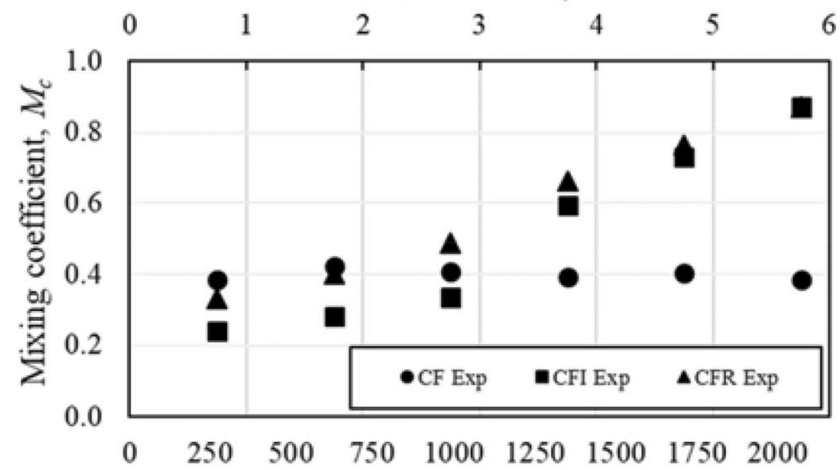

Angle of revolution, $\theta\left[^{\circ}\right]$

$$
R e=200
$$

Number of turns, $n$

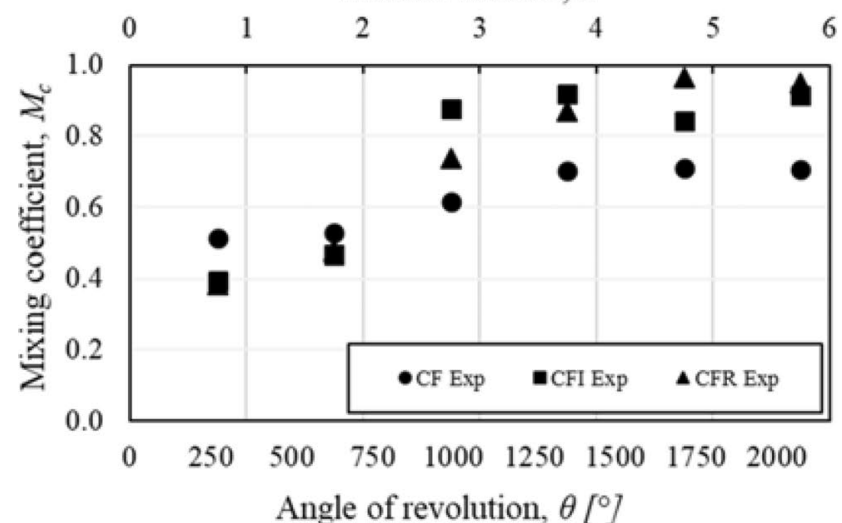

$$
R e=1000
$$

Number of turns, $n$

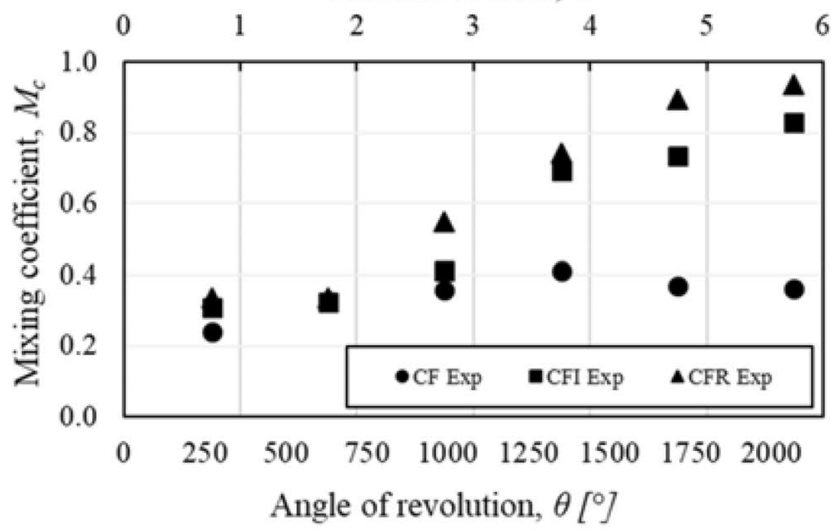

$R e=100$

Number of turns, $n$

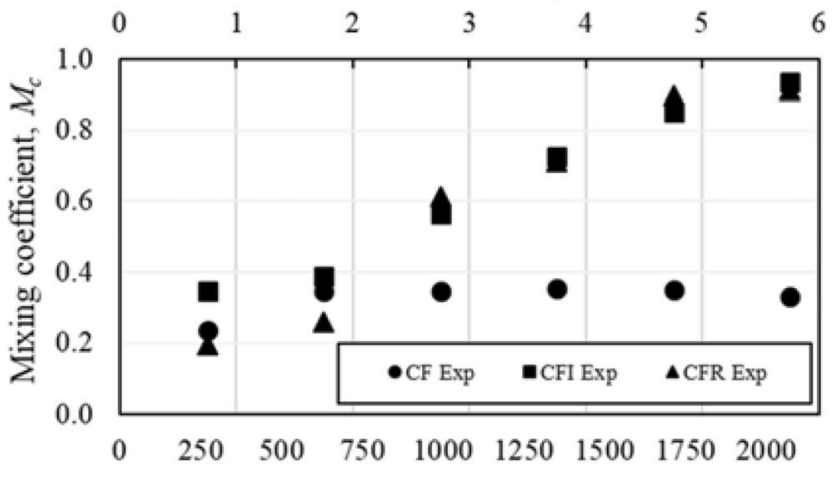

Angle of revolution, $\theta\left[{ }^{\circ}\right]$

$$
R e=500
$$

Fig. 10 Experimental results for the axial development of the mixing coefficient in the 6-turn configurations 


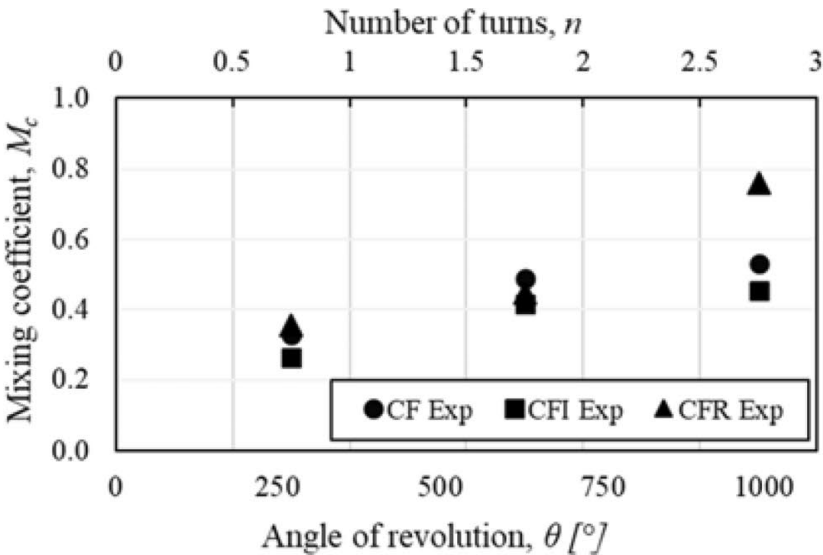

$$
\operatorname{Re}=50
$$

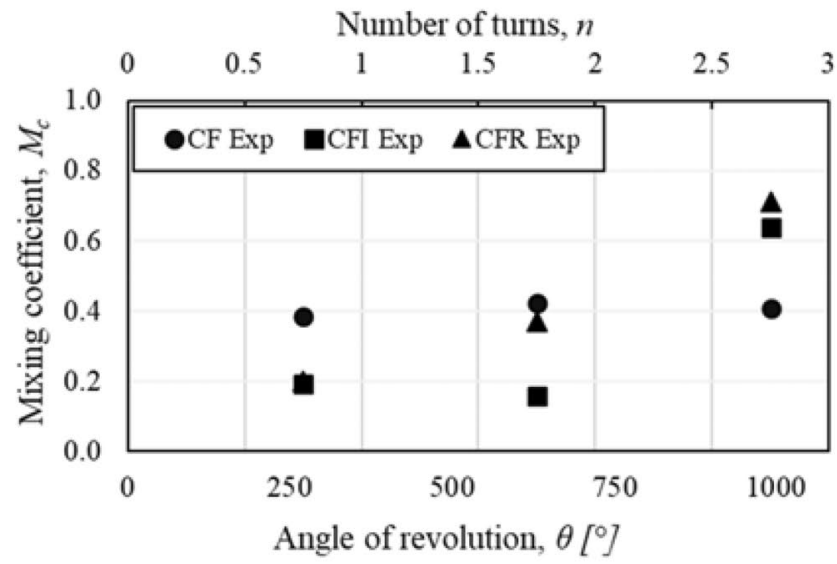

$$
R e=200
$$

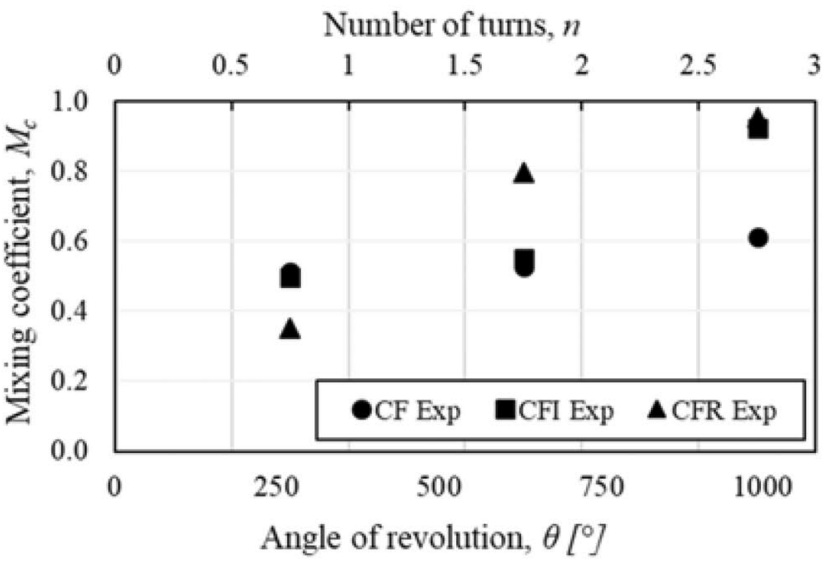

$$
R e=1000
$$

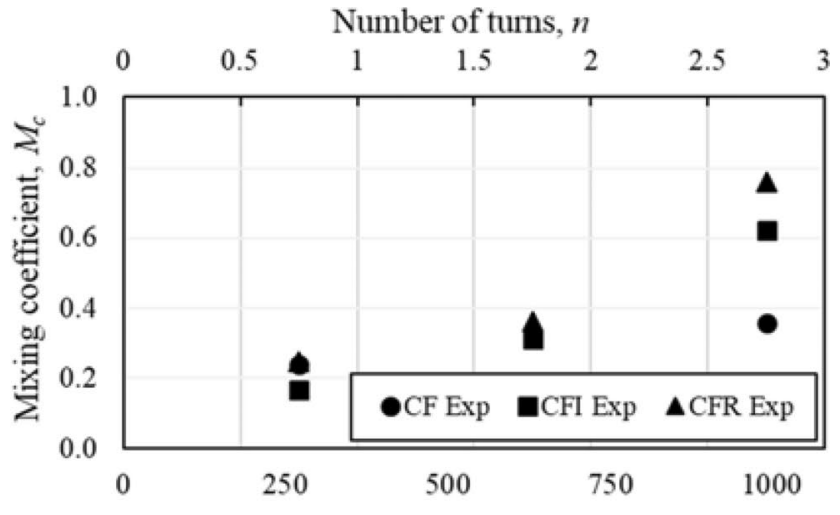

Angle of revolution, $\theta\left[{ }^{\circ}\right]$

$$
R e=100
$$

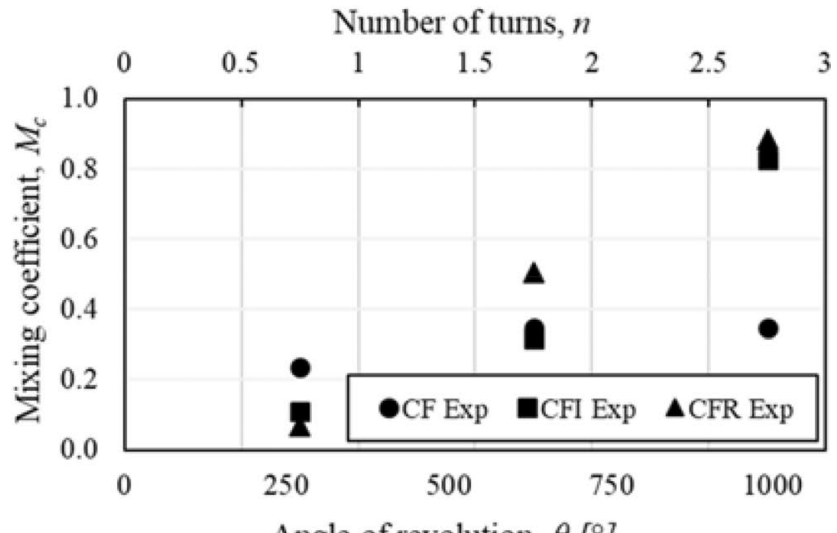

$R e=500$

Fig. 11 Experimental results for the axial development of the mixing coefficient in the 3-turn configurations

\section{Validation of numerical calculations}

The experimental results shown above, have been used to validate numerical calculations using the same geometry and inlet conditions.

\subsection{Numerical method}

Similar to our previous studies (Khot et al. 2019; Mansour et al. 2017, 2019, 2020c), the CFD code Star-CCM + was used to simulate the fluid flow and the mixing characteristics of the experimental geometries. The species transport model 
was used to calculate the mixing between the two liquids (liquid 1: L1 and liquid 2: L2). In this model, an additional (scalar) transport equation is solved to determine the mass fraction (concentration) of each liquid along with the continuity and the momentum equations (Mansour et al. 2017). A steady-state solver was applied for all simulations, since the preliminary tests confirmed a steady behaviour of the mass fraction of both liquids in all coiled configurations for the considered Reynolds number range. Additionally, a laminar setup was selected, since the critical Reynolds number for the helical coils are much higher than the considered Reynolds number values (Cioncolini and Santini 2006; Mishra and Gupta 1979; Schmidt 1967; Srinivasan et al. 1968).

The segregated solver with the SIMPLE algorithm was employed to treat the coupling between velocity and pressure, since it requires less memory than the coupled solver. The second-order upwind scheme was employed as a discretization scheme to determine the convective fluxes in the transport equations for both the flow and concentration fields. The second-order upwind scheme combines the benefits of the central differencing and the first-order upwind schemes. Additionally, the second-order upwind scheme has considerably lower numerical diffusion compared to the first-order scheme (Bailey 2017; Tu et al. 2018; Versteeg and Malalasekera 2007). No-slip boundary condition and the zero-derivative conditions for scalars were imposed along all walls. At the outlet surface, a uniform pressure outlet boundary condition was used.

In the simulations, the physical properties of the two liquids (L1 and L2) were considered as those used in the experiments (water), with a density of $\rho=998.23 \mathrm{~kg} / \mathrm{m}^{3}$ and a dynamic viscosity of $\eta=0.001003 \mathrm{~Pa} \cdot \mathrm{s}$. A Schmidt number value of $S c=1000$ was set in the setup, which corresponds typically to water. The solution was considered as converged when the normalized residuals went below $10^{-7}$ for the continuity and momentum equations, and below $10^{-5}$ for the scalar species transport equation. Further details concerning the governing equations can be found in (Mansour et al. 2017).

\subsection{Geometry and mesh}

Figure 12 shows the considered simulation domains of all different helical configurations. All dimensions are kept identical to the experiments, even the ellipsoidal section of the experimental helical tube (see Fig. 13). Additionally, in a similar way to the experiments, a Y-connector is used at the inlet to allow for realistic inlet conditions for each liquid. Further, to ensure accurate mixing behaviour, a Hagen-Poiseuille parabolic velocity profile was set for each branch of the Y-connector to eliminate the use of the uniform velocity boundary conditions, which results in unrealistic mixing as discussed in (Mansour et al. 2017). For spatial discretization, hexahedral elements were always generated, since it

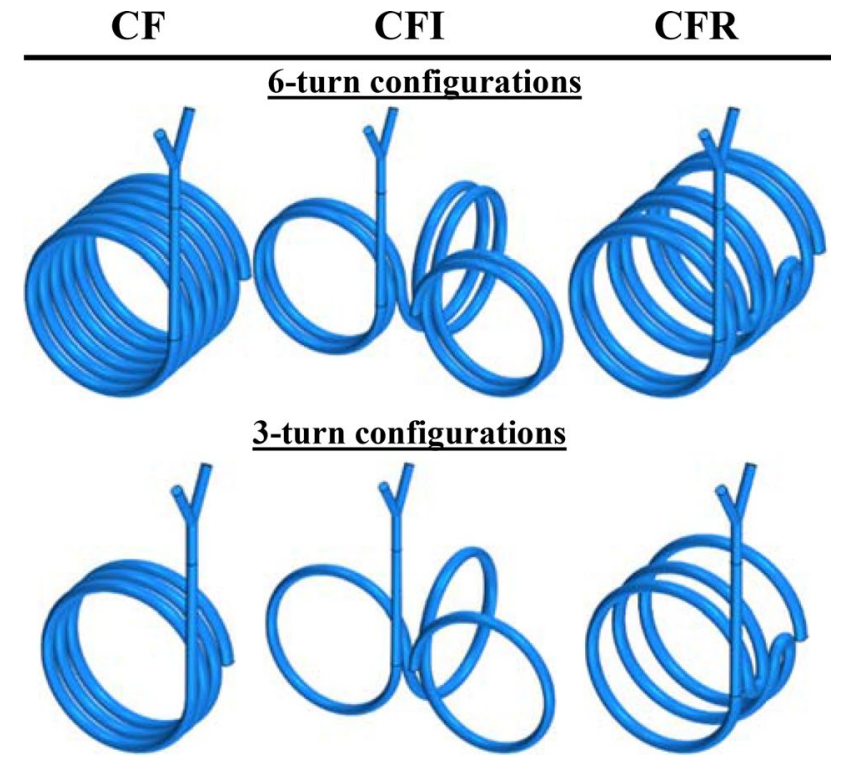

Fig. 12 Different simulation domains considered

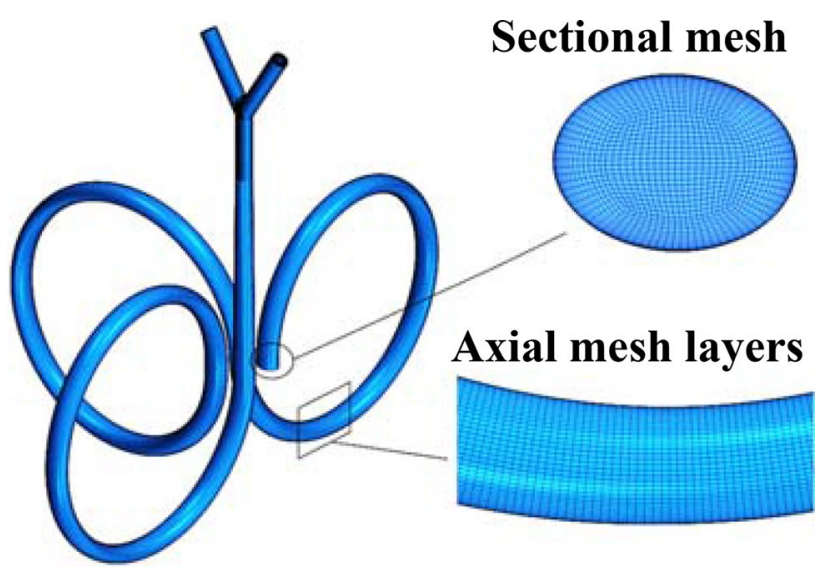

Fig. 13 Details of the mesh structure

was recently shown that hexahedral elements lead to minimum numerical errors compared to polyhedral or tetrahedral elements (Mansour et al. 2020a). A total number of 7.7 and 4.8 million cells were used for the 6-turn and 3-turn configurations, respectively.

A sample view of the mesh structure is shown in Fig. 13 for the 3-turn configuration. As shown, the mesh is always aligned with the main flow direction to minimize the numerical diffusion. Additionally, a typical refinement is applied near the wall to ensure accurate resolving of the boundary layer flow. The employed numerical model and mesh have been validated against measurements of (Mori and Nakayama 1965) and successfully used in the aforementioned previous studies. 

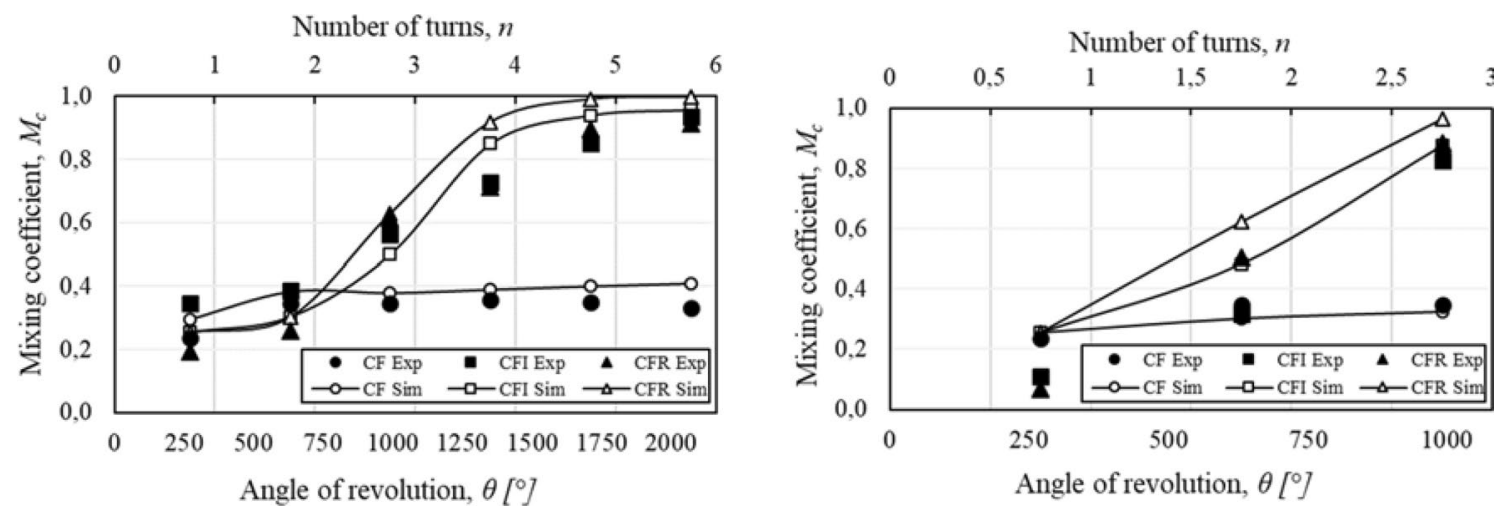

Fig. 14 Comparison of experimental and numerical results for the axial development of the mixing coefficient in the 6-turn (left) and 3-turn configurations (right) at $R e=500$

\subsection{Comparison of experimental and numerical results}

Exemplarily, the comparison of the experimentally and numerically obtained mixing coefficients and mass fractions for $R e=500$ and all six configurations can be found in Fig. 14 and Fig. 15 as a function of the angle of revolution $\theta$ and the position in the reactor. In Fig. 14, the experimental results are represented as plain symbols, the numerical results as lines with corresponding empty symbols at the measurement position.

The overall comparison of the results shows a rather good accordance between the numerical and experimental results, especially for the straight coils. Also, the mixing coefficients and mass fractions in the first and last turns at the outlet are well reproduced by the calculations. Only for the central turns of both CFI and CFR configurations, the numerical calculations predict higher mixing coefficients than the experimental results. To explain this, it has to be kept in mind, that the numerical calculations consider a smooth, continuous tube, while in the experimental tube sleeves and reversers lead to small steps between the assembled tube parts and, especially for the CFI and CFR, small deviations of the geometry after each flow reversing, while the geometry in the calculation stays constant. Also, given the accuracy of the measurements, which lies around $10 \%$, most of the deviations between numerical simulations and experiments are covered by this measurement error.

In Fig. 16 the mixing coefficients in the last turn of the CFR, that showed the best mixing qualities throughout the experiments, are represented for experiments and numerical calculations as a function of the Reynolds number. It is very interesting to compare the results of the 6-turn configuration (left in Fig. 16) and those of the 3-turn configurations (right in Fig. 16). The doubling of reactor length leads only to a small increase in mixing performance (smaller than measurement accuracy). The influence of the Reynolds number on the mixing coefficient at the outlet is relatively small, especially for the 6-turn CFR. This reactor configuration offers the best mixing characteristics with mixing coefficients in the range of 0.8 to nearly 1.0. The highest mixing coefficient has been attained by both CFR reactors at $R e=1000$ with a value of 0.95 in the experiment.

\section{Conclusions}

In this experimental study, mixing in three different helically coiled configurations at two different lengths has been analyzed by laser-induced fluorescence in the laminar flow regime. A resorufin-water solution has been used to visualize and quantify mixing in a straight horizontal helical coil, a coiled flow inverter, and a novel coiled flow reverser. The results obtained by PLIF, have been used for the validation of numerical calculations using the same geometry and inlet conditions as in the experiments. All results show, that an early flow redirection is a key feature for good radial mixing performances. For this reason, the coiled flow inverter and coiled flow reverser exhibit much better overall mixing performance, than the straight coil. Also, the 6-turn configurations achieve only slightly better mixing, than the 3-turn reactors, underlying the importance of early flow redirection for increased heat and mass transfer characteristics of coiled reactors working in the laminar regime. 


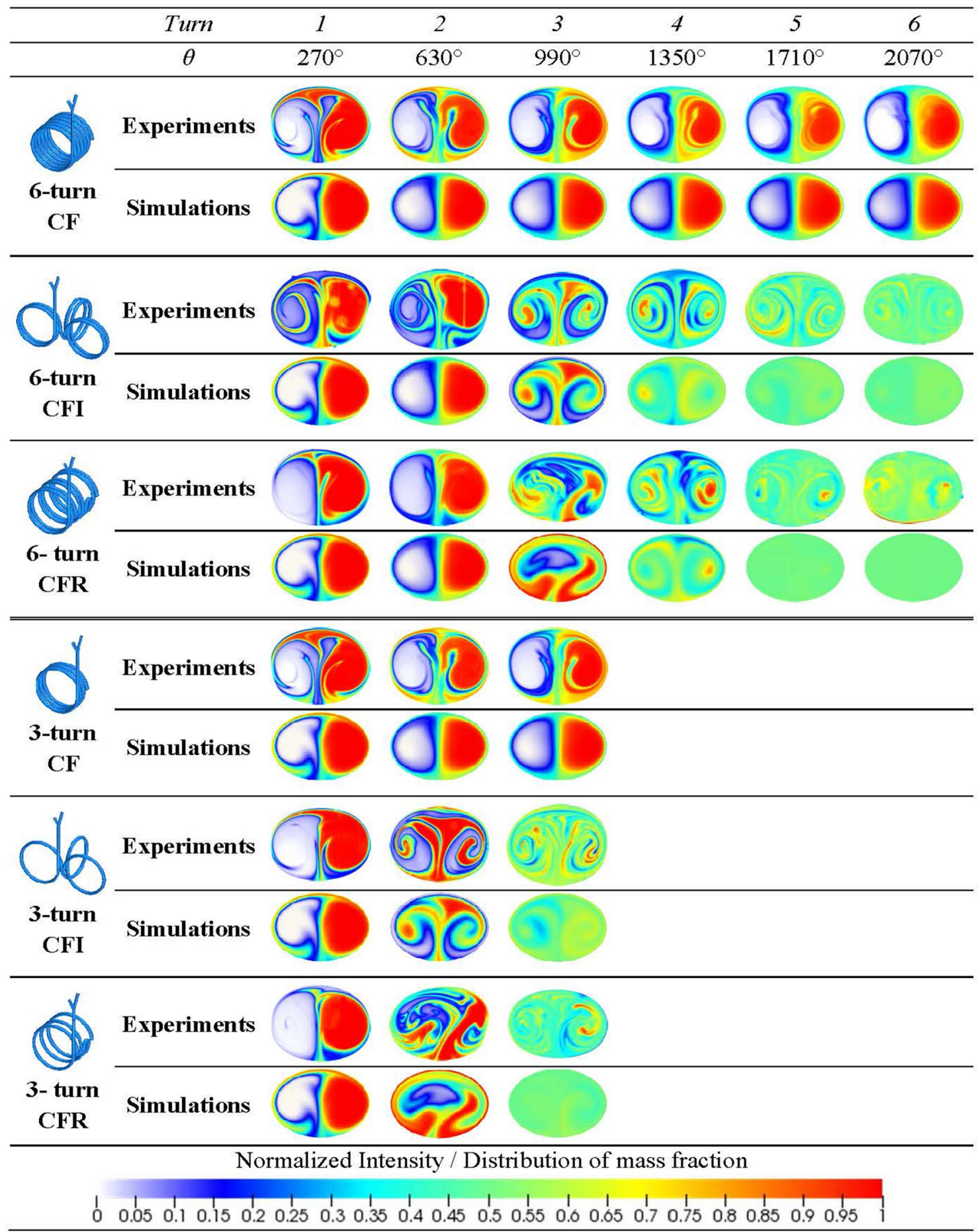

Fig. 15 Comparison of experimental and numerical results for the axial development of mass fractions in all six configurations at $R e=500$ 

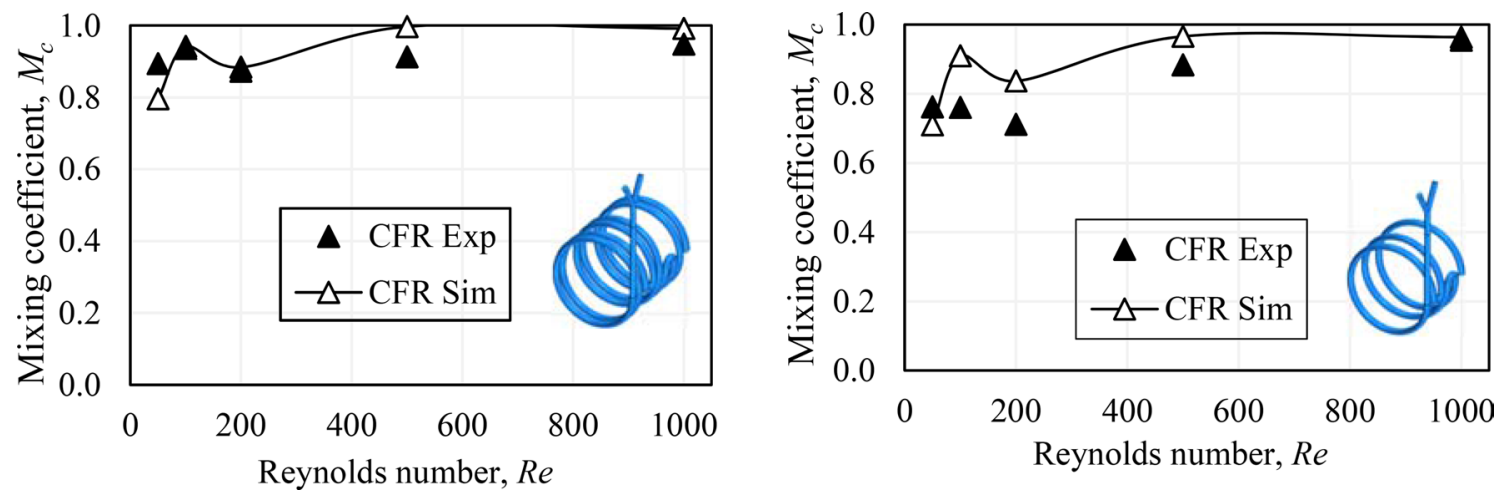

Fig. 16 Experimental and numerical results for the mixing coefficient in the last turn of the coiled flow reverser (left: 6-turn CFR, right: 3-turn CFR)

Acknowledgements Open Access funding provided by Projekt DEAL. Authors would like to thank the Deutsche Forschungsgemeinschaft (DFG, German Research Foundation) - TRR 63 "Integrated Chemical Processes in Liquid Multiphase Systems" (subproject B1)—56091768 (Gefördert durch die Deutsche Forschungsgemeinschaft (DFG)_TRR 63 "Integrierte chemische Prozesse in flüssigen Mehrphasensystemen" (Teilprojekt B1) - 56091768). Our student Conrad Müller is acknowledged for his help during experiments, as well as Prafull Khot for doing some of the calculations.

Open Access This article is licensed under a Creative Commons Attribution 4.0 International License, which permits use, sharing, adaptation, distribution and reproduction in any medium or format, as long as you give appropriate credit to the original author(s) and the source, provide a link to the Creative Commons licence, and indicate if changes were made. The images or other third party material in this article are included in the article's Creative Commons licence, unless indicated otherwise in a credit line to the material. If material is not included in the article's Creative Commons licence and your intended use is not permitted by statutory regulation or exceeds the permitted use, you will need to obtain permission directly from the copyright holder. To view a copy of this licence, visit http://creativecommons.org/licenses/by/4.0/.

\section{References}

Alam A, Kim K-Y (2012) Analysis of mixing in a curved microchannel with rectangular grooves. Chem Eng J 181-182:708-716

Austin LR, Seader JD (1973) Fully developed viscous flow in coiled circular pipes. AIChE J 19:85-94. https://doi.org/10.1002/ aic.690190113

Bailey RT (2017) Managing false diffusion during second-order upwind simulations of liquid micromixing. Int J Numer Meth Fl 83:940-959

Castelain C, Legentilhomme P (2006) Residence time distribution of a purely viscous non-Newtonian fluid in helically coiled or spatially chaotic flows. Chem Eng J 120:181-191

Cioncolini A, Santini L (2006) An experimental investigation regarding the laminar to turbulent flow transition in helically coiled pipes. Exp Thermal Fluid Sci 30:367-380. https://doi.org/10.1016/j. expthermflusci.2005.08.005

Dong L, Shufen Z (2014) Numerical and experimental investigation of the effect of geometrical parameters on the performance of a contraction-expansion helical mixer. Int J Chem React Eng $12: 465-475$
Gelfgat AY, Yarin AL, Bar-Yoseph PZ (2003) Dean vortices-induced enhancement of mass transfer through an interface separating two immiscible liquids. Phys Fluids 15:330-347. https://doi. org/10.1063/1.1532732

Hajmohammadi MR, Eskandari H, Saffar-Avval M, Campo A (2013) A new configuration of bend tubes for compound optimization of heat and fluid flow. Energy 62:418-424

Ito $\mathrm{H}$ (1959) Friction factors for turbulent flow in curved pipes. J Basic Eng 81:123-134

Jiang F, Drese KS, Hardt S, Küpper M, Schönfeld F (2004) Helical flows and chaotic mixing in curved micro channels. AIChE J 50:2297-2305

Khot P, Mansour M, Thévenin D, Nigam KDP, Zähringer K (2019) Improving the mixing characteristics of coiled configurations by early flow inversion. Chem Eng Res Des 146:324-335. https://doi. org/10.1016/j.cherd.2019.04.016

Kockmann N (2020) Design and operation of a tubular reactor with narrow residence time distribution. Chem-Ing-Tech 92:685-691. https://doi.org/10.1002/cite.202000028

Kováts P, Zähringer K (2018) Detailed measurement of oxygen mass transfer in a bubble column-application of a new fluorescent dye. In: 19th International Symposium on the Application of Laser and Imaging Techniques to Fluid Mechanics, pp. 3.16.11, Lisbon, Portugal

Kováts P, Pohl D, Thévenin D, Zähringer K (2018) Optical determination of oxygen mass transfer in a helically-coiled pipe compared to a straight horizontal tube. Chem Eng Sci 190:273-285. https:// doi.org/10.1016/j.ces.2018.06.029

Kumar V, Aggarwal M, Nigam KDP (2006) Mixing in curved tubes. Chem Eng Sci 61:5742-5753. https://doi.org/10.1016/j. ces.2006.04.040

Kumar V, Mridha M, Gupta AK, Nigam KDP (2007) Coiled flow inverter as a heat exchanger. Chem Eng Sci 62:2386-2396. https ://doi.org/10.1016/j.ces.2007.01.032

Kurt SK, Gelhausen MG, Kockmann N (2015a) Axial dispersion and heat transfer in a milli/microstructured coiled flow inverter for narrow residence time distribution at laminar flow. Chem Eng Technol 38:1122-1130. https://doi.org/10.1002/ceat.201400515

Kurt SK, Nigam KDP, Kockmann N (2015b) Two-phase flow and mass transfer in helical capillary flow reactors with alternating bends. In: ASME 2015 International Technical Conference and Exhibition on Packaging and Integration of Electronic and Photonic Microsystems, InterPACK 2015, collocated with the ASME 2015 13th International Conference on Nanochannels, Microchannels, and Minichannels 
Kushwaha N, Kumawat TC, Nigam KDP, Kumar V (2020) Heat transfer and fluid flow characteristics for Newtonian and nonNewtonian fluids in a tube-in-tube helical coil heat exchanger. Ind Eng Chem Res 59:3972-3984. https://doi.org/10.1021/acs. iecr.9b07044

Lasbet Y, Auvity B, Castelain C, Peerhossaini H (2007) Thermal and hydrodynamic performances of chaotic mini-channel: application to the fuel cell cooling. Heat Transf Eng 28:795-803

Liu L, Li L, Ding Z, Ma R, Yang Z (2005) Mass transfer enhancement in coiled hollow fiber membrane modules. J Membr Sci 264:113121. https://doi.org/10.1016/j.memsci.2005.04.035

López-Guajardo E, Ortiz-Nadal E, Montesinos-Castellanos A, Nigam KDP (2017) Coiled flow inverter as a novel alternative for the intensification of a liquid-liquid reaction. Chem Eng Sci 169:179185. https://doi.org/10.1016/j.ces.2017.01.016

Mandal MM, Aggarwal P, Nigam KDP (2011) Liquid-liquid mixing in coiled flow inverter. Ind Eng Chem Res 50:13230-13235. https:// doi.org/10.1021/ie2002473

Mansour M, Liu Z, Janiga G, Nigam KDP, Sundmacher K, Thévenin D, Zähringer K (2017) Numerical study of liquid-liquid mixing in helical pipes. Chem Eng Sci 172:250-261. https://doi. org/10.1016/j.ces.2017.06.015

Mansour M, Janiga G, Nigam KDP, Thévenin D, Zähringer K (2018) Numerical study of heat transfer and thermal homogenization in a helical reactor. Chem Eng Sci 177:369-379. https://doi. org/10.1016/j.ces.2017.11.031

Mansour M, Thévenin D, Nigam KDP, Zähringer K (2019) Generallyvalid optimal Reynolds and Dean numbers for efficient liquidliquid mixing in helical pipes. Chem Eng Sci 201:382-385. https ://doi.org/10.1016/j.ces.2019.03.003

Mansour M, Khot P, Kováts P, Thévenin D, Zähringer K, Janiga G (2020) Impact of computational domain discretization and gradient limiters on CFD results concerning liquid mixing in a helical pipe. Chem Eng J 383:123121. https://doi.org/10.1016/j. cej.2019.123121

Mansour M, Khot P, Thévenin D, Nigam KDP, Zähringer K (2020) Optimal Reynolds number for liquid-liquid mixing in helical pipes. Chem Eng Sci 214:114522. https://doi.org/10.1016/j. ces.2018.09.046

Mansour M, Thévenin D, Zähringer K (2020) Numerical study of flow mixing and heat transfer in helical pipes, coiled flow inverters and a novel coiled configuration. Chem Eng Sci 221:115690. https:// doi.org/10.1016/j.ces.2020.115690

Mei M, Felis F, Hébrard G, Dietrich N, Loubière K (2020) Hydrodynamics of gas-liquid slug flows in a long in-plane spiral shaped milli-reactor. Theor Found Chem Eng 54:25-47. https://doi. org/10.1134/S0040579520010169

Mishra P, Gupta S (1979) Momentum transfer in curved pipes. 1. Newtonian fluids. Ind Eng Chem Process Design Dev 18:130-137

Mori Y, Nakayama W (1965) Study of forced convective heat transfer in curved pipes (1st report, laminar region). Int J Heat Mass Transf 8:67-82. https://doi.org/10.1016/0017-9310(67)90182-2

Moulin P, Rouch JC, Serra C, Clifton MJ, Aptel P (1996) Mass transfer improvement by secondary flows: dean vortices in coiled tubular membranes. J Membr Sci 114:235-244. https://doi. org/10.1016/0376-7388(95)00323-1

Mridha M, Nigam KDP (2008a) Coiled flow inverter as an inline mixer. Chem Eng Sci 63:1724-1732. https://doi.org/10.1016/j. ces. 2007.10 .028

Mridha M, Nigam KDP (2008b) Numerical study of turbulent forced convection in coiled flow inverter. Chem Eng Process 47:893-905. https://doi.org/10.1016/j.cep.2007.02.026

Naphon P, Wongwises S (2006) A review of flow and heat transfer characteristics in curved tubes. Renew Sustain Energy Rev 10:463-490. https://doi.org/10.1016/j.rser.2004.09.014
Ndiaye D (2017) Transient model of a refrigerant-to-water helically coiled tube-in-tube heat exchanger with corrugated inner tube. Appl Therm Eng 112:413-423

Rainieri S, Bozzoli F, Pagliarini G (2012) Experimental investigation on the convective heat transfer in straight and coiled corrugated tubes for highly viscous fluids: preliminary results. Int $\mathbf{J}$ Heat Mass Transf 55:498-504

Rennie TJ, Raghavan VGS (2005) Experimental studies of a doublepipe helical heat exchanger. Exp Therm Fluid Sci 29:919-924

Saffari H, Moosavi R (2014) Numerical study of the influence of geometrical characteristics of a vertical helical coil on a bubbly flow. $\mathrm{J}$ Appl Mech Tech Phys 55:957-969. https://doi.org/10.1134/S0021 894414060066

Saxena AK, Nigam K (1984) Coiled configuration for flow inversion and its effect on residence time distribution. AIChE J 30:363-368. https://doi.org/10.1002/aic.690300303

Schmidt EF (1967) Heat transfer and pressure loss in pipe coils. Chem Ing Tec 39:781-789

Schönfeld F, Hardt S (2004) Simulation of helical flows in microchannels. AIChE J 50:771-778

Sedahmed GH, Shemilt LW, Wong F (1985) Natural convection mass transfer characteristics of rings and helical coils in relation to their use in electrochemical reactor design. Chem Eng Sci 40:11091114. https://doi.org/10.1016/0009-2509(85)85069-7

Sharma L, Nigam KDP, Roy S (2017) Single phase mixing in coiled tubes and coiled flow inverters in different flow regimes. Chem Eng Sci 160:227-235. https://doi.org/10.1016/j.ces.2016.11.034

Srinivasan PS, Nandapurkar SS, Holland FA (1968) Pressure drop and heat transfer in coils. Chem Eng 218:113-119

Tohidi A, Ghaffari H, Nasibi H, Mujumdar AS (2015) Heat transfer enhancement by combination of chaotic advection and nanofluids flow in helically coiled tube. Appl Therm Eng 86:91-105. https:// doi.org/10.1016/j.applthermaleng.2015.04.043

Tu J, Yeoh GH, Liu C (2018) Computational fluid dynamics: a practical approach. Butterworth-Heinemann, Oxford

Vanka SP, Luo G, Winkler CM (2004) Numerical study of scalar mixing in curved channels at low Reynolds numbers. AIChE J 50:2359-2368

Vashisth S, Nigam KDP (2009) Prediction of flow profiles and interfacial phenomena for two-phase flow in coiled tubes. Chem Eng Process 48:452-463. https://doi.org/10.1016/j.cep.2008.06.006

Vashisth S, Kumar V, Nigam KDP (2008) A review on the potential applications of curved geometries in process industry. Ind Eng Chem Res 47:3291-3337. https://doi.org/10.1021/ie701760h

VDI e.V. (2013) VDI-Wärmeatlas, 11th edn. Springer, Berlin. https:// doi.org/10.1007/978-3-642-19981-3

Versteeg HK, Malalasekera W (2007) An introduction to computational fluid dynamics: the finite volume method. Pearson education. ISBN 9780131274983

Wang Y, Alvarado JL, Terrell W (2018) Thermal and flow characteristics of helical coils with reversed loops. Int J Heat Mass Transf 126:670-680. https://doi.org/10.1016/j.ijheatmasstrans fer.2018.02.110

Yasuo M, Wataru N (1965) Study on forced convective heat transfer in curved pipes. Int J Heat Mass Transf 8:67-82. https://doi. org/10.1016/0017-9310(65)90098-0

Zachár A (2010) Analysis of coiled-tube heat exchangers to improve heat transfer rate with spirally corrugated wall. Int J Heat Mass Transf 53:3928-3939

Publisher's Note Springer Nature remains neutral with regard to jurisdictional claims in published maps and institutional affiliations. 